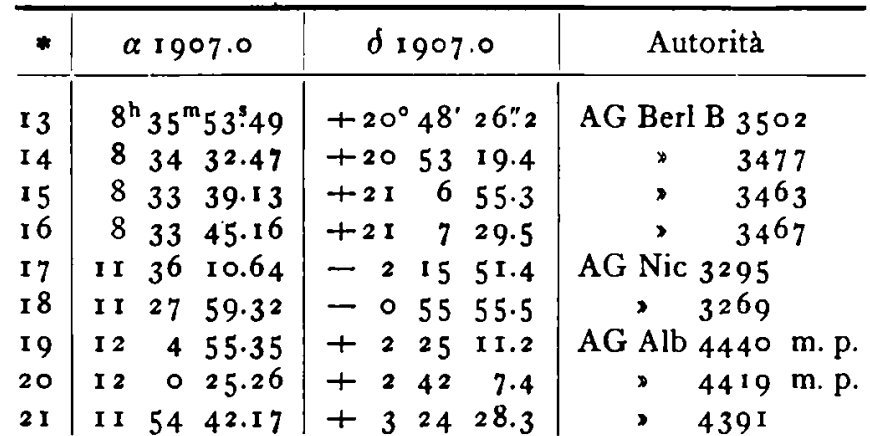

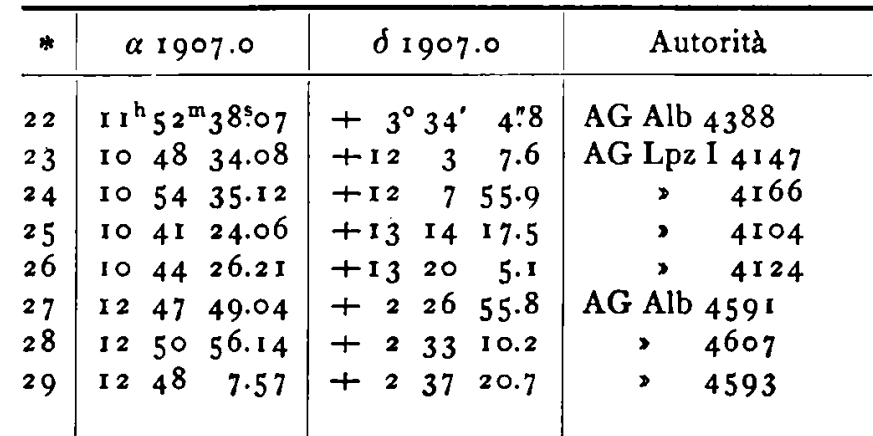

Confronto delle osservazioni colle effemeridi nel senso $(\mathrm{O}-\mathrm{C})$.

\begin{tabular}{l|l|l}
\hline $\mathrm{I} 907$ & $\Delta \alpha$ & $\Delta \delta$ \\
\hline
\end{tabular}

(8) Flora.

[Effemeride Downing, Monthly Notices, 66. p. 548.]

\begin{tabular}{r|r|r} 
Gen. 10 & -10.03 & +19.9 \\
17 & -9.92 & +19.2 \\
17 & -9.97 & +17.2 \\
18 & -10.02 & +20.3 \\
18 & -10.00 & +19.2
\end{tabular}

Padova, 24 Aprile 1907.

\begin{tabular}{|c|c|c|c|}
\hline I 907 & & $\Delta \alpha$ & $\Delta \delta$ \\
\hline \multicolumn{4}{|c|}{$\begin{array}{l}\text { (79) Eurynome. } \\
\text { [B. A. J. I 909.] }\end{array}$} \\
\hline \multirow[t]{4}{*}{ Marz. } & 4 & -0.75 & + I 2.0 \\
\hline & 5 & -0.77 & +10.8 \\
\hline & 15 & -0.65 & +10.5 \\
\hline & 16 & $-0.8 \mathrm{I}$ & $+\mathrm{I} 2.5$ \\
\hline \multicolumn{4}{|c|}{$\begin{array}{l}\text { (82) Alkmene. } \\
\text { [B. A. J. r gog.] }\end{array}$} \\
\hline Marz. & 5 & +1.35 & $-I I .2$ \\
\hline & 9 & +1.35 & $-I 3 \cdot I$ \\
\hline
\end{tabular}

9) Eurynome

A. J. I 909 .

\begin{tabular}{r|c|c}
\hline 1907 & $\Delta \alpha$ & $\Delta \delta$ \\
\hline Marz.18 & +1.55 & -12.0 \\
20 & +1.64 & -10.8 \\
21 & +1.59 & -10.7 \\
\multicolumn{3}{c}{ (68) Leto. } \\
[B. A. J. 1909.] \\
Marz.1 2 & +2.75 & -39.6 \\
1 2 & +2.91 & -40.0 \\
13 & +2.96 & -40.4 \\
I3 & +2.97 & -40.1
\end{tabular}

G. A. Favaro.

\title{
Photographische Beobachtungen von kleinen Planeten
}

mit dem Bredichinschen Astrographen zu Pulkowo von $N$. Liapin.

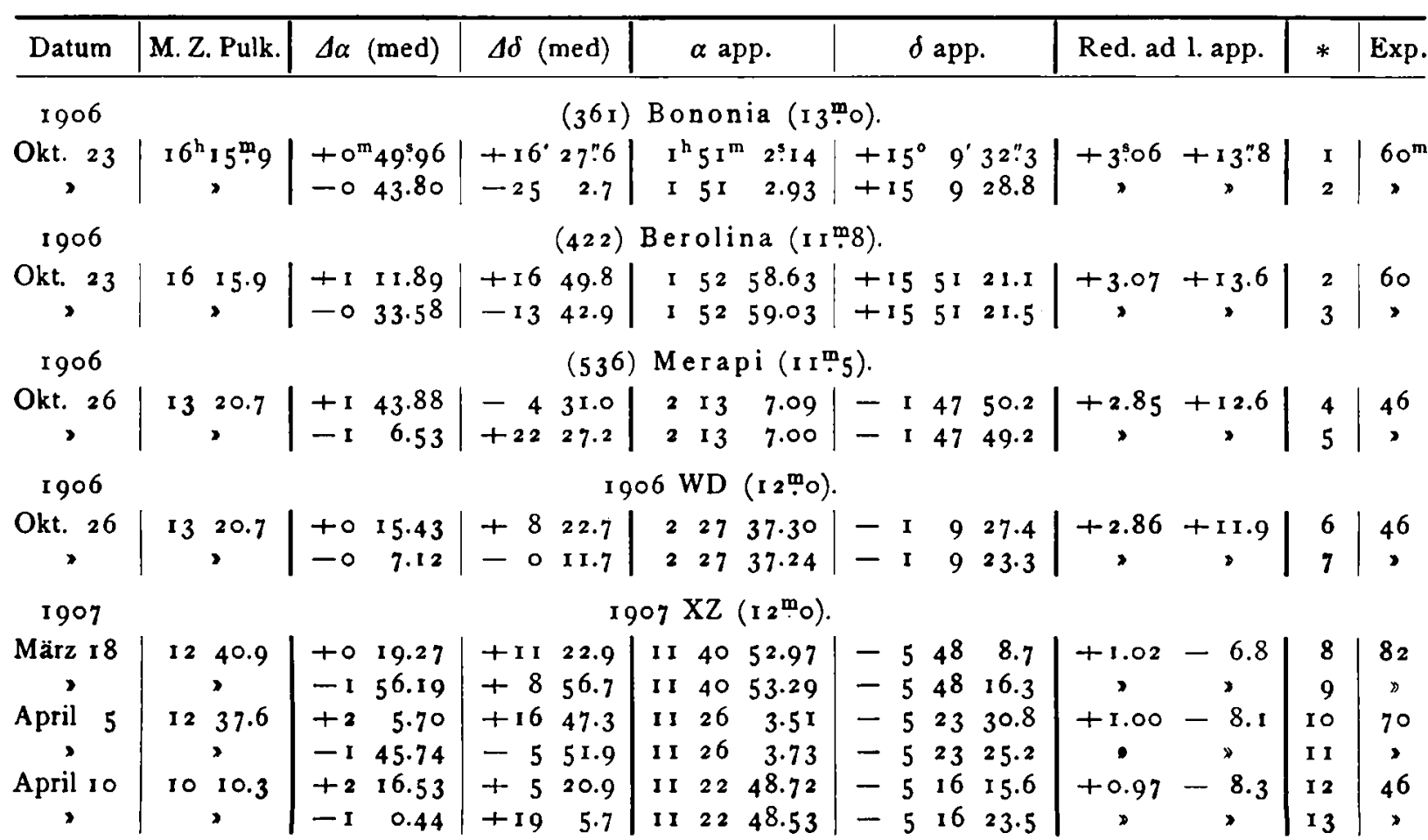


Mittlere Örter der Vergleichsterne für den Anfang des Beobachitungjahres.

\begin{tabular}{|c|c|c|c|c|}
\hline * & $\alpha$ & & $\delta$ & Autorität \\
\hline $\mathbf{I}$ & $1^{\mathrm{h}} 50^{\mathrm{m}} 9^{8}: 12$ & $+14^{\circ}$ & $52^{\prime} 50^{\prime \prime} 9$ & $A G L_{p z} I_{572}$ \\
\hline 2 & I 5143.67 & +15 & $34 \quad 17.7$ & AG Berl A 554 \\
\hline 3 & I $53 \quad 29.54$ & +16 & 450.8 &,$\quad 564$ \\
\hline 4 & 21120.36 & $-\mathbf{I}$ & 4331.8 & $\begin{array}{l}1 / 2 \text { (AG Strb } 545+\text { AG } \\
\left.\text { Nic } 45^{\circ}\right)\end{array}$ \\
\hline 5 & $2 \quad 14 \quad 10.68$ & -2 & 1029.0 & $\begin{array}{l}1 / 2 \text { (AG Strb } 560+\text { War } \\
380)\end{array}$ \\
\hline 6 & 2719.01 & $-I$ & 2.0 & AG Nic 5 I 2 \\
\hline
\end{tabular}

\begin{tabular}{|c|c|c|c|c|c|c|}
\hline • & $\alpha$ & & & $\delta$ & & Autorität \\
\hline 7 & $2^{\mathrm{h}} 27^{\mathrm{m}} 4 \mathrm{I}^{3} \cdot 50$ & 一 & $1^{\circ}$ & & $23: 5$ & AG Nic 5 I 6 \\
\hline 8 & II 4032.68 & - & 55 & 59 & 24.8 & AG Strb 4405 \\
\hline 9 & I I 4248.46 & - & 5 & 57 & 6.2 & 4414 \\
\hline Io & I $2356.8 \mathrm{i}$ & - & 5 & 40 & I 0.0 & 4319 \\
\hline I I & I I 2748.47 & - & 5 & I 7 & 25.2 & $434^{\circ}$ \\
\hline 12 & I I $20 \quad 31.22$ & - & 5 & $2 I$ & 28.2 & $43 \circ 5$ \\
\hline I 3 & I I 2348.00 & - & 53 & 35 & 20.9 & $43 I 7$ \\
\hline
\end{tabular}

Pulkowo, 1907 Mai 7 .

N. Liapin.

\section{Photographische 'Aufnahmen des Kometen $1907 \mathrm{~d}$.}

Mit provisorisch aufgestelltem Äquatoreal und $3^{1 / 4}$-z. $\mid=2^{\circ}$. 3) PW. $=264^{\circ} 3, \mathrm{~L} .=10^{\circ}$. 4) PW. $=266: 8$, Portrătobjektiv (Petzval-Ross) ist es uns gelungen, den Ko- $\mathrm{L} .=10^{\circ}$. Annähernd $4^{\circ}$ vom Kern mit vorigem Strahl meten $1907 \mathrm{~d}$ in der ersten Halfte August einigemal aufzunehmen. Die Platte Nr. 3081907 Aug. 14, Expositionszeit $13^{\mathrm{h}} 57^{\mathrm{m}}-15^{\mathrm{h}} \circ^{\mathrm{m}}$, zeigt folgende Details:

Schweif $10^{\circ}$ lang (bis zum Plattenrande) hat in der ersten Hälfte eine ausgeprägt fächerartige Struktur. Die Positionswinkel (Äqu. 1855) und Längen einzelner Strahlen sind: I) $\mathrm{PW} .=25698, \mathrm{~L} .=2^{\circ}$. 2) $\mathrm{PW} .=25898, \mathrm{~L}$.

Privatsternwarte Žalov b. Ondřejov (Böhmen).
\[ \text { Ephemeride des Planeten (471) Papagena. } \] bogenartig verbunden (anderer Strahl?). 5) Fällt bis zu einer Entfernung von fast $2^{\circ}$ vom Kern mit dem vorigen zusammen, dann gebogen und von der Richtung der Schweifachse merklich abgeneigt. $\mathrm{PW} .=269^{\circ} 2, \mathrm{~L} .=10^{\circ}$. 6) $\mathrm{PW} .=273^{\circ} 3, \mathrm{~L} .=2{ }^{\circ}$; erscheint doppelt. Die Platte laßt noch feinere Struktur vermuten.

Aus den von mir abgeleiteten und in den Astr. Nachr. Nr. 4199 publizierten Elementen habe ich die folgende Aufsuchungsephemeride für $\mathbf{I}^{\mathrm{h}}{ }^{\mathrm{h}}$ M. Z. Berlin gerechnet.

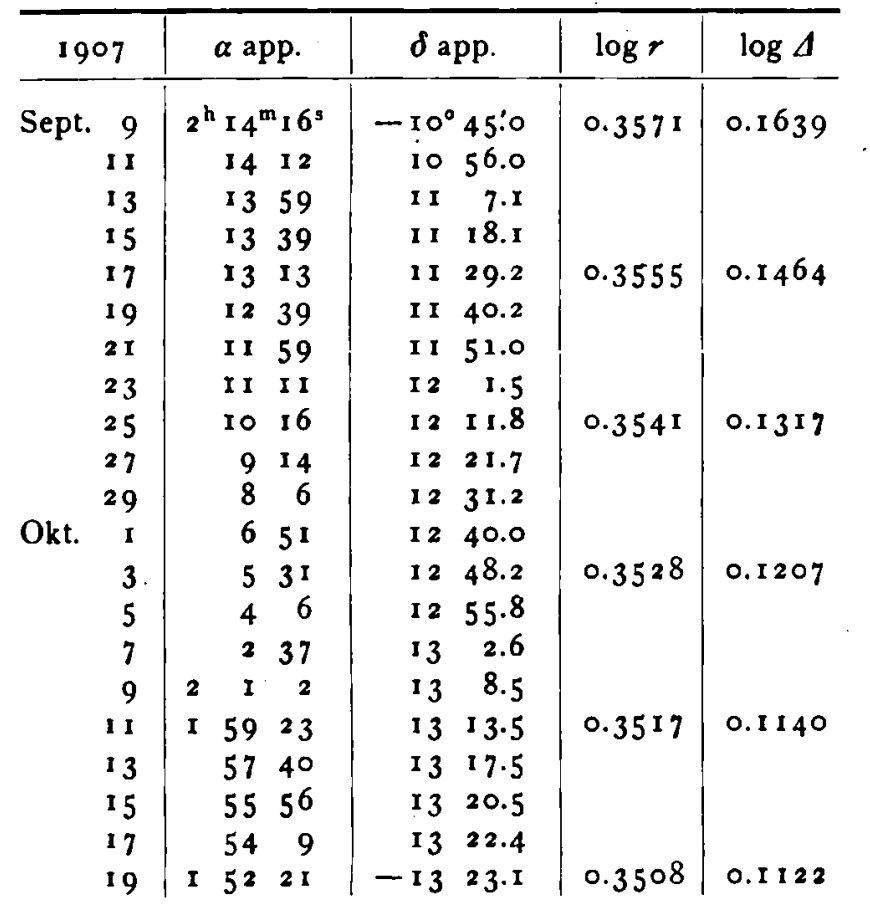

\begin{tabular}{|c|c|c|c|c|c|}
\hline \multicolumn{2}{|c|}{1907} & $\alpha$ app. & $\delta$ app. & $\log r$ & $\log 4$ \\
\hline Okt. & 19 & $I^{k} 5^{2^{m}} 2 I^{3}$ & $-I 3^{\circ}=3^{\prime} \mathrm{I}$ & 0.3508 & 0.1122 \\
\hline & 21 & $50 \quad 32$ & I 322.6 & & \\
\hline & 23 & $\begin{array}{lll}48 & 43\end{array}$ & I 321.0 & & \\
\hline & 25 & $4^{6} 55$ & I 318.0 & & \\
\hline 8 & 27 & 458 & I. $3 \quad 13.9$ & 0.3500 & 0.1154 \\
\hline & 29 & 4321 & I 38.4 & & \\
\hline & 31 & $41 \quad 37$ & I $3 \quad 1.7$ & & \\
\hline Nov. & 2 & 3956 & I 253.7 & & \\
\hline & 4 & 38 I9 & 1244.6 & 0.3494 & 0.1234 \\
\hline & 6 & $3^{6} 4^{6}$ & I 234.0 & & \\
\hline & 8 & $35 \quad 17$ & I $2 \quad 23.3$ & & \\
\hline & 10 & $33 \quad 53$ & I $2 \quad 9.5$ & & \\
\hline & 12 & $3^{2} 36$ & II 55.5 & $0.34^{8} 9$ & 0.1357 \\
\hline & I 4 & $\begin{array}{ll}3 & 2\end{array}$ & I I 40.3 & & \\
\hline & 16 & $\begin{array}{ll}30 & 17\end{array}$ & I I 24.2 & & \\
\hline & 18 & 2917 & I I 7.0 & & \\
\hline & 20 & 2824 & IO 48.8 & 0.3486 & 0.1516 \\
\hline & 22 & $273^{8}$ & IO 29.7 & & \\
\hline & 24 & $265^{8}$ & I $0 \quad 9.8$ & & \\
\hline & 26 & 2625 & $949 . I$ & & \\
\hline & 28 & I $255^{8}$ & 927.6 & $0.34^{8} 5$ & 0.1702 \\
\hline
\end{tabular}

Lichtstärke des Planeten bei der Opposition $8^{\mathrm{m}} \cdot 5$.

Der Planet kommt in das Perihel Nov. 30 und ist wegen seiner großen Annäherung an die Erde sehr günstig zu beobachten. Akurejri, Island, 1907 Aug. 15. Gustaf Strömberg, Assistent an der Sternwarte in Stockholm. 